\title{
Overpartitions with restricted odd differences
}

\author{
Kathrin Bringmann* \\ Mathematical Institut \\ University of Cologne \\ 50931 Cologne, Germany \\ kbringma@math.uni-koeln.de \\ Jeremy Lovejoy \\ CNRS, LIAFA \\ Université Paris Diderot - Paris 7 \\ 75205 Paris Cedex 13, France \\ lovejoy@math.cnrs.fr \\ Jehanne Dousse $^{\dagger}$ \\ LIAFA \\ Université Paris Diderot - Paris 7 \\ 75205 Paris Cedex 13, France \\ jehanne.dousse@liafa.univ-paris-diderot.fr \\ Karl Mahlburg $\ddagger$ \\ Department of Mathematics \\ Louisiana State University \\ Baton Rouge, LA 70802, USA \\ mahlburg@math.lsu.edu
}

Submitted: May 12, 2015; Accepted: Jul 17, 2015; Published: Jul 31, 2015

Mathematics Subject Classifications: 05A17, 11P82

\begin{abstract}
We use $q$-difference equations to compute a two-variable $q$-hypergeometric generating function for overpartitions where the difference between two successive parts may be odd only if the larger part is overlined. This generating function specializes in one case to a modular form, and in another to a mixed mock modular form. We also establish a two-variable generating function for the same overpartitions with odd smallest part, and again find modular and mixed mock modular specializations. Applications include linear congruences arising from eigenforms for 3-adic Hecke operators, as well as asymptotic formulas for the enumeration functions. The latter are proven using Wright's variation of the circle method.
\end{abstract}

Keywords: overpartitions; $q$-difference equations; mixed mock modular forms; Wright's circle method

*The research of the first author was supported by the Alfried Krupp Prize for Young University Teachers of the Krupp foundation and the research leading to these results has received funding from the European Research Council under the European Union's Seventh Framework Programme (FP/2007-2013) / ERC Grant agreement n. 335220 - AQSER

$\dagger$ Most of this research was conducted while the second author was visiting the University of Cologne funded by the Krupp foundation

‡The first and fourth authors thank the Alexander von Humboldt Foundation 


\section{Introduction and statement of results}

An overpartition of $n$ is a partition of $n$ in which the final occurrence of a number may be overlined. Let $\bar{p}(n)$ denote the number of overpartitions of $n$. For example, $\bar{p}(4)=14$, the 14 overpartitions of 4 being

$$
\begin{gathered}
4, \overline{4}, 3+1, \overline{3}+1,3+\overline{1}, \overline{3}+\overline{1}, 2+2,2+\overline{2} \\
2+1+1, \overline{2}+1+1,2+1+\overline{1}, \overline{2}+1+\overline{1}, 1+1+1+1,1+1+1+\overline{1}
\end{gathered}
$$

Next, let $\bar{t}(n)$ denote the number of overpartitions where $(i)$ the difference between two successive parts may be odd only if the larger part is overlined, and $(i i)$ if the smallest part is odd then it is overlined. Let $\bar{s}(n)$ denote the number of overpartitions counted by $\bar{t}(n)$ but with odd smallest part. Thus $\bar{t}(4)=8$ and $\bar{s}(4)=4$, the 8 overpartitions counted by $\bar{t}(4)$ being

$$
4, \overline{4}, 3+\overline{1}, \overline{3}+\overline{1}, 2+2,2+\overline{2}, \overline{2}+1+\overline{1}, 1+1+1+\overline{1}
$$

and the 4 overpartitions counted by $\bar{s}(4)$ being

$$
3+\overline{1}, \overline{3}+\overline{1}, \overline{2}+1+\overline{1}, 1+1+1+\overline{1} .
$$

The first goal of this paper is to determine $q$-hypergeometric generating functions for $\bar{t}(m, n)$ and $\bar{s}(m, n)$, the number of overpartitions counted by $\bar{t}(n)$ (resp. $\bar{s}(n))$ having $m$ parts. We employ the usual notation $\left(n \in \mathbb{N}_{0} \cup\{\infty\}\right)$,

$$
(a)_{n}:=(a ; q)_{n}:=(1-a)(1-a q) \cdots\left(1-a q^{n-1}\right) .
$$

Theorem 1. We have the following identities:

$$
\begin{aligned}
\sum_{m, n \geqslant 0} \bar{t}(m, n) x^{m} q^{n} & =\frac{(-x q)_{\infty}}{(x q)_{\infty}}\left(1+\sum_{n \geqslant 1} \frac{\left(-q^{3} ; q^{3}\right)_{n-1}(-x)^{n} q^{n}}{(-q)_{n-1}\left(q^{2} ; q^{2}\right)_{n}}\right), \\
\sum_{m, n \geqslant 1} \bar{s}(m, n) x^{m} q^{n} & =\sum_{n \geqslant 1} \frac{\left(q^{3} ; q^{3}\right)_{n-1} x^{n} q^{n}}{(q)_{n-1}\left(q^{2} ; q^{2}\right)_{n}} .
\end{aligned}
$$

The second identity follows from a straightforward combinatorial argument, but the first is more subtle and our proof depends on showing that both sides satisfy a certain $q$ difference equation. Note that the positivity of the coefficients on the right-hand side of (1) is not immediately apparent.

When $x=1$ in (1) or -1 in (2), then we have a modular form, and when $x=-1$ in (1) or 1 in (2), then we have the product of a modular form and a mock theta function, a so-called mixed mock modular form (see [11]). More precisely, define the mock theta functions $\bar{\gamma}(q)$ and $\bar{\chi}(q)$ by

$$
\bar{\gamma}(q):=\sum_{n \geqslant 0} \frac{(-1)_{n}(q)_{n} q^{\left(\begin{array}{c}
n+1 \\
2
\end{array}\right)}}{\left(q^{3} ; q^{3}\right)_{n}}
$$


and

$$
\bar{\chi}(q):=\sum_{n \geqslant 0} \frac{(-1)_{n}(-q)_{n} q^{\left(\begin{array}{c}
n+1 \\
2
\end{array}\right)}}{\left(-q^{3} ; q^{3}\right)_{n}} .
$$

(That these are mock theta functions follows from work of the first and the third authors [5].) Let $\bar{t}_{ \pm}(n)$ (resp. $\bar{s}_{ \pm}(n)$ ) denote the number of overparititions counted by $\bar{t}(n)$ (resp. $\bar{s}(n))$ with largest part even (resp. odd).

Corollary 2. We have

$$
\begin{aligned}
\sum_{n \geqslant 0} \bar{t}(n) q^{n} & =\frac{\left(q^{3} ; q^{3}\right)_{\infty}}{(q)_{\infty}\left(q^{2} ; q^{2}\right)_{\infty}}, \\
\sum_{n \geqslant 0}\left(\bar{t}_{+}(n)-\bar{t}_{-}(n)\right) q^{n} & =\frac{\left(-q^{3} ; q^{3}\right)_{\infty}}{(-q)_{\infty}^{3}} \bar{\chi}(q), \\
1+3 \sum_{n \geqslant 1}\left(\bar{s}_{+}(n)-\bar{s}_{-}(n)\right) q^{n} & =\frac{\left(-q^{3} ; q^{3}\right)_{\infty}}{(-q)_{\infty}^{3}}, \\
1+3 \sum_{n \geqslant 1} \bar{s}(n) q^{n} & =\frac{\left(q^{3} ; q^{3}\right)_{\infty}}{(q)_{\infty}\left(q^{2} ; q^{2}\right)_{\infty}} \bar{\gamma}(q) .
\end{aligned}
$$

Equation (6) combined with work of the first and the third author on overpartitions and class numbers $[6,7]$ implies that the generating function for $\bar{t}_{+}(n)-\bar{t}_{-}(n)$ is an eigenform modulo 3 for the weight $3 / 2$ Hecke operators. This is recorded below along with a congruence for $\bar{t}(n)$ modulo 3. Even though the congruence for $\bar{t}(n)$ is immediate from (5), it would be interesting to see if it can be deduced from an explicit 3-fold symmetry for the overpartitions counted by $\bar{t}(n)$.

\section{Corollary 3.}

1. For a prime $\ell \neq 2,3$, and $n \geqslant 0$, we have

$$
\begin{aligned}
\bar{t}_{+}\left(\ell^{2} n\right) & +\left(\left(\frac{-n}{\ell}\right)-\ell-1\right) \bar{t}_{+}(n)+\ell \bar{t}_{+}\left(\frac{n}{\ell^{2}}\right) \\
& \equiv \bar{t}_{-}\left(\ell^{2} n\right)+\left(\left(\frac{-n}{\ell}\right)-\ell-1\right) \bar{t}_{-}(n)+\ell \bar{t}_{-}\left(\frac{n}{\ell^{2}}\right) \quad(\bmod 3) .
\end{aligned}
$$

2. For $n \geqslant 1$, we have

$$
\bar{t}(n) \equiv \begin{cases}(-1)^{k+1} \quad(\bmod 3) & \text { if } n=k^{2} \\ 0 \quad(\bmod 3) & \text { otherwise }\end{cases}
$$

Our final results give asymptotic formulas for $\bar{s}(n)$ and $\bar{t}_{+}(n)-\bar{t}_{-}(n)$, which are the cases from Corollary 2 in which the generating functions are mixed mock modular forms. 
Theorem 4. As $n \rightarrow \infty$, we have

$$
\begin{aligned}
\bar{s}(n) & \sim \frac{\sqrt{21}}{36 n} e^{\frac{\pi \sqrt{7 n}}{3}}, \\
\bar{t}_{+}(n)-\bar{t}_{-}(n) & \sim(-1)^{n} \frac{\sqrt{3}}{18 n^{\frac{3}{4}}} e^{\frac{2 \pi \sqrt{n}}{3}} .
\end{aligned}
$$

Remark. Since (5) and (7) are (up to rational q-powers) weakly holomorphic modular forms of non-positive weight, Rademacher and Zuckerman's famous refinement of the Hardy-Ramanujan Circle Method applies [9, 12, 13]. These results allow one to use the cuspidal principal parts in order to calculate exact formulas for the coefficients. In fact, one finds that $\bar{t}(n)$ is asymptotically equivalent to $(11)$ and $\bar{s}_{+}(n)-\bar{s}_{-}(n)$ is asymptotically equivalent to (12).

We have highlighted the two cases in Theorem 4 because the Hardy-RamanujanRademacher Circle Method does not generally apply to mixed mock modular forms. Instead we use Wright's Circle Method [15], which uses a single "Major Arc" centered around a dominant cusp to derive an asymptotic expansion for the coefficients. In most examples in the literature this cusp is $q=1$, but for (12) the Major Arc is instead centered at $q=-1$.

The rest of the paper is organized as follows. In the next section we prove Theorem 1 using analytic and combinatorial arguments. In Section 3 we then apply $q$-series identities to establish Corollaries 2 and 3. Section 4 outlines the proof of Theorem 4 using Wright's Circle Method. We conclude in Section 5 by briefly tying the present work to other recent studies of mixed mock modular $q$-series and $q$-difference equations.

\section{Combinatorial recurrences and the proof of Theorem 1}

In this section we use the analytic and combinatorial theory of $q$-difference equations to prove Theorem 1 . We begin by considering (1). Define $\bar{H}(x ; q)$ by

$$
\bar{H}(x ; q):=\sum_{m, n \geqslant 0} \bar{t}(m, n) x^{m} q^{n} .
$$

Now, if $\lambda$ is an overpartition counted by $\bar{t}(m, n)$, then its smallest part is either $\overline{1}$ or at least 2 . In the first case, we may remove the $\overline{1}$ along with any other $1 \mathrm{~s}$ in $\lambda$ and then subtract 1 from each remaining part to obtain an overpartition counted by $\bar{t}(m-a, n-m)$, where $a \geqslant 1$ is the number of 1 s occurring in $\lambda$. In the second case, we may remove any $2 \mathrm{~s}$ in $\lambda$ and then subtract 2 from each remaining part to obtain an overpartition counted by $\bar{t}(m-b, n-2 m)$, where $b \geqslant 0$ is the number of 2 s occurring in $\lambda$. Note that if the smallest part in this new partition is odd, then it must have correspondingly occurred as an overlined part in $\lambda$. In other words, we have

$$
\bar{H}(x ; q)=\frac{x q}{1-x q} \bar{H}(x q ; q)+\frac{1+x q^{2}}{1-x q^{2}} \bar{H}\left(x q^{2} ; q\right) .
$$


Together with the fact that $\bar{H}(0 ; q)=1$, this uniquely defines $\bar{H}(x ; q)$.

We claim that the right-hand side of (1) also satisfies (13) with the same initial condition. To see this, first define

$$
\bar{K}(x ; q)=\bar{K}(x):=1+\sum_{n \geqslant 1} \frac{\prod_{j=1}^{n-1}\left(1-q^{j}+q^{2 j}\right)}{\left(q^{2} ; q^{2}\right)_{n}} x^{n} q^{n} .
$$

We have that

$$
\begin{aligned}
\bar{K}\left(x q^{2}\right) & =1-\sum_{n \geqslant 1} \frac{x^{n} q^{n} \prod_{j=1}^{n-1}\left(1-q^{j}+q^{2 j}\right)}{\left(q^{2} ; q^{2}\right)_{n}}\left(1-q^{2 n}-1\right) \\
& =\bar{K}(x)-\sum_{n \geqslant 1} \frac{x^{n} q^{n} \prod_{j=1}^{n-1}\left(1-q^{j}+q^{2 j}\right)}{\left(q^{2} ; q^{2}\right)_{n-1}} \\
& =\bar{K}(x)-\sum_{n \geqslant 0} \frac{x^{n+1} q^{n+1} \prod_{j=1}^{n-1}\left(1-q^{j}+q^{2 j}\right)\left(1-q^{n}+q^{2 n}\right)}{\left(q^{2} ; q^{2}\right)_{n}} \\
& =\bar{K}(x)-x q \bar{K}(x)+x q \bar{K}(x q)-x q \bar{K}\left(x q^{2}\right)
\end{aligned}
$$

so that

$$
\bar{K}(x)=-\frac{x q}{1-x q} \bar{K}(x q)+\frac{1+x q}{1-x q} \bar{K}\left(x q^{2}\right) .
$$

Now, the right-hand side of $(1)$ is $\bar{G}(x ; q)$, where

$$
\bar{G}(x ; q)=\bar{G}(x):=\frac{(-x q)_{\infty}}{(x q)_{\infty}} \bar{K}(-x),
$$

and applying (14) to $\bar{G}(x ; q)$ gives

$$
\bar{G}(x ; q)=\frac{x q}{1-x q} \bar{G}(x q ; q)+\frac{1+x q^{2}}{1-x q^{2}} \bar{G}\left(x q^{2} ; q\right) .
$$

Comparing (13) and (15) and noting the initial condition $\bar{G}(0 ; q)=1$, yields (1).

Next we consider (2). Here we require the notion of the conjugate of an overpartition, which is obtained by reading the columns of the Ferrers diagram (an overlined part is designated by a mark at the end of a row). The right-hand side of (2) is

$$
\sum_{n \geqslant 1} \frac{\left(q^{3} ; q^{3}\right)_{n-1} x^{n} q^{n}}{(q)_{n-1}\left(q^{2} ; q^{2}\right)_{n}}=\sum_{m \geqslant 1} \frac{x^{m} q^{m}}{1-q^{2 m}} \prod_{j=1}^{m-1}\left(\frac{q^{j}}{1-q^{j}}+\frac{1}{1-q^{2 j}}\right) .
$$

Here the $m$ th summand is the generating function for overpartitions where the largest part $m$ occurs overlined and an odd number of times, while each part less than $m$ occurs an even number of times if it does not occur overlined. Conjugating we obtain an overpartition counted by $\bar{s}(m, n)$. This is illustrated in Figure 1. This establishes (2) and completes the proof of Theorem 1. 

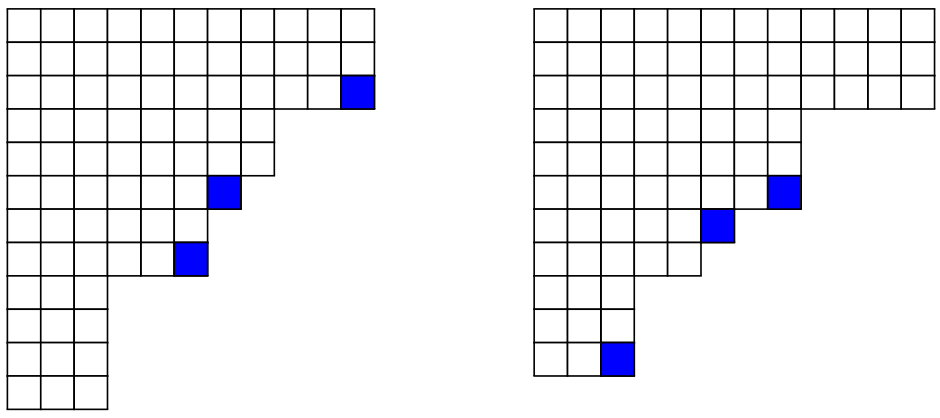

Figure 1: The overpartition $(11,11, \overline{11}, 8,8, \overline{7}, 6, \overline{6}, 3,3,3,3)$ and its conjugate $(12,12,12,8,8, \overline{8}, \overline{6}, 5,3,3, \overline{3})$.

\section{Proof of Corollaries 2 and 3}

In this section we use $q$-series identities to obtain the generating functions for overpartitions with restricted odd differences in Corollary 2. We require the $q$-Gauss summation formula [8, Equation (1.5.1)],

$$
\sum_{n \geqslant 0} \frac{(a)_{n}(b)_{n}(c / a b)^{n}}{(c)_{n}(q)_{n}}=\frac{(c / a)_{\infty}(c / b)_{\infty}}{(c)_{\infty}(c / a b)_{\infty}},
$$

along with a certain ${ }_{3} \phi_{2}$ transformation [8, Appendix III, Equation (III.10)],

$$
\sum_{n \geqslant 0} \frac{(a q / b c)_{n}(d)_{n}(e)_{n}}{(q)_{n}(a q / b)_{n}(a q / c)_{n}}\left(\frac{a q}{d e}\right)^{n}=\frac{(a q / d)_{\infty}(a q / e)_{\infty}(a q / b c)_{\infty}}{(a q / b)_{\infty}(a q / c)_{\infty}(a q / d e)_{\infty}} \sum_{n \geqslant 0} \frac{(a q / d e)_{n}(b)_{n}(c)_{n}}{(q)_{n}(a q / d)_{n}(a q / e)_{n}}\left(\frac{a q}{b c}\right)^{n} .
$$

Proof of Corollary 2. Setting $x=1$ in (1) and applying (16) with $a=1 / b=-\zeta_{3}$ (where $\zeta_{3}:=e^{\frac{2 \pi i}{3}}$ ) and $c=-q$, we obtain

$$
\begin{aligned}
\sum_{n \geqslant 0} \bar{t}(n) q^{n} & =\frac{(-q)_{\infty}}{(q)_{\infty}} \sum_{n \geqslant 0} \frac{\left(-\zeta_{3}\right)_{n}\left(-\zeta_{3}^{-1}\right)_{n}(-q)^{n}}{(q)_{n}(-q)_{n}} \\
& =\frac{(-q)_{\infty}}{(q)_{\infty}} \times \frac{\left(\zeta_{3} q\right)_{\infty}\left(\zeta_{3}^{-1} q\right)_{\infty}}{(-q)_{\infty}^{2}} \\
& =\frac{\left(q^{3} ; q^{3}\right)_{\infty}}{(q)_{\infty}\left(q^{2} ; q^{2}\right)_{\infty}},
\end{aligned}
$$

which proves (5).

For the next case we set $x=-1$ in (1) and invoke (17) with $a=-b=1, d=1 / e=-\zeta_{3}$, and $c \rightarrow \infty$, which yields 


$$
\begin{aligned}
\sum_{n \geqslant 0}\left(\bar{t}_{+}(n)-\bar{t}_{-}(n)\right) q^{n} & =\frac{(q)_{\infty}}{(-q)_{\infty}} \sum_{n \geqslant 0} \frac{\left(-\zeta_{3}\right)_{n}\left(-\zeta_{3}^{-1}\right)_{n} q^{n}}{(q)_{n}(-q)_{n}} \\
& =\frac{(q)_{\infty}}{(-q)_{\infty}} \times \frac{\left(-\zeta_{3} q\right)_{\infty}\left(-\zeta_{3}^{-1} q\right)_{\infty}}{(q)_{\infty}(-q)_{\infty}} \sum_{n \geqslant 0} \frac{(-1)_{n} q^{\left(\begin{array}{c}
n+1 \\
2
\end{array}\right)}}{\left(-\zeta_{3} q\right)_{n}\left(-\zeta_{3}^{-1} q\right)_{n}} \\
& =\frac{\left(-q^{3} ; q^{3}\right)_{\infty}}{(-q)_{\infty}^{3}} \bar{\chi}(q) .
\end{aligned}
$$

The final two equations in the statement of the corollary are proven similarly. For $(7)$ we set $x=-1$ in (2) and apply (16) with $a=1 / b=\zeta_{3}$ and $c=-q$, whereas for (8) we set $x=1$ in (2) and appeal to (17) in the case $a=-b=1, d=1 / e=\zeta_{3}$, and $c \rightarrow \infty$.

We remark that (5) can also be established by a simple combinatorial argument, as in the proof of (2). Indeed, conjugating an overpartition $\lambda$ counted by $\bar{t}(n)$ gives an overpartition with the property that if $\bar{m}$ doesn't occur, then $m$ occurs an even number of times. Hence

$$
\sum_{n \geqslant 0} \bar{t}(n) q^{n}=\prod_{m \geqslant 1}\left(\frac{q^{m}}{\left(1-q^{m}\right)}+\frac{1}{\left(1-q^{2 m}\right)}\right)=\frac{\left(q^{3} ; q^{3}\right)_{\infty}}{\left(q^{2} ; q^{2}\right)_{\infty}(q)_{\infty}} .
$$

This argument easily generalizes. Let $\bar{t}^{(k)}(n)$ denote the number of overpartitions of $n$ where $(i)$ consecutive parts differ by a multiple of $(k+1)$ unless the larger of the two is overlined, and $(i i)$ the smallest part is overlined unless it is divisible by $k+1$. Then we have

$$
\sum_{n \geqslant 0} \bar{t}^{(k)}(n) q^{n}=\prod_{m \geqslant 1}\left(\frac{q^{m}}{\left(1-q^{m}\right)}+\frac{1}{\left(1-q^{k m}\right)}\right)=\frac{\left(q^{k+1} ; q^{k+1}\right)_{\infty}}{\left(q^{k} ; q^{k}\right)_{\infty}(q)_{\infty}}
$$

We close this section by determining the behavior of these generating functions modulo 3 , which is achieved by relating them to previously studied Hecke eigenforms.

Proof of Corollary 3. Reading (6) modulo 3, we have

$$
\sum_{n \geqslant 0}\left(\bar{t}_{+}(n)-\bar{t}_{-}(n)\right) q^{n} \equiv \sum_{n \geqslant 0} \frac{(-1)_{n} q^{\left(\begin{array}{c}
n+1 \\
2
\end{array}\right)}}{(-q)_{n}^{2}} \quad(\bmod 3) .
$$

We recall from Proposition 5.1 of [7] that if $\bar{\alpha}(n)$ is defined by

$$
\sum_{n \geqslant 0} \bar{\alpha}(n) q^{n}:=\sum_{n \geqslant 0} \frac{(-1)_{n} q^{\left(\begin{array}{c}
n+1 \\
2
\end{array}\right)}}{(-q)_{n}^{2}}
$$

then

$$
\bar{\alpha}\left(\ell^{2} n\right)+\left(\frac{-n}{\ell}\right) \bar{\alpha}(n)+\ell \bar{\alpha}\left(\frac{n}{\ell^{2}}\right)=(\ell+1) \bar{\alpha}(n) .
$$


This proves (9).

To show (10), we read (5) modulo 3, obtaining

$$
\sum_{n \geqslant 0} \bar{t}(n) q^{n} \equiv \frac{(q)_{\infty}^{2}}{\left(q^{2} ; q^{2}\right)_{\infty}}=1+2 \sum_{n \geqslant 1}(-1)^{n} q^{n^{2}} \quad(\bmod 3) .
$$

The equality is Gauss' identity [1, Equation (2.2.12)].

\section{Wright's Circle Method and the proof of Theorem 4}

In this section we apply Wright's Circle Method [15] to prove Theorem 4. As is typical of the Circle Method, Wright's approach recovers the coefficients of a generating series by applying Cauchy's Theorem, with the main asymptotic contribution coming from a single Major Arc (for our two results, this is centered around $q=1$ and $q=-1$, respectively). For notational convenience we recall (6) and (8) and write

$$
\begin{aligned}
& f_{1}(q):=1+3 \sum_{n \geqslant 1} \bar{s}(n) q^{n}=\frac{\left(q^{3} ; q^{3}\right)_{\infty}}{(q)_{\infty}\left(q^{2} ; q^{2}\right)_{\infty}} \bar{\gamma}(q), \\
& f_{2}(q):=\sum_{n \geqslant 0}\left(\bar{t}_{+}(n)-\bar{t}_{-}(n)\right) q^{n}=\frac{\left(-q^{3} ; q^{3}\right)_{\infty}}{(-q)_{\infty}^{3}} \bar{\chi}(q) .
\end{aligned}
$$

\subsection{Analytic behavior of $f_{1}(q)$}

We begin with $\bar{s}(n)$, as the asymptotic analysis is analogous to Wright's original examples, which were also centered around $q=1$. Throughout the section we therefore use the standard parameterization $q=e^{2 \pi i \tau}$, where $\tau=x+i y$ and $y>0$. Recalling (3), we use an alternative expression for the mock theta function, which follows from equation (1.3) in [5], namely

$$
\bar{\gamma}(q)=\frac{(-q)_{\infty}}{(q)_{\infty}}\left(1+6 \sum_{k \geqslant 1} \frac{(-1)^{k} q^{k^{2}+k}}{\left(1-\zeta_{3} q^{k}\right)\left(1-\zeta_{3}^{-1} q^{k}\right)}\right)
$$

with $\zeta_{3}:=e^{\frac{2 \pi i}{3}}$.

We show that the overall asymptotic behavior of $f_{1}(q)$ is largely controlled by the singularities of the infinite product from (18), so the dominant pole is at $q=1$. The following result describes the behavior of $f_{1}(q)$ in a neighborhood of $q=1$ and gives a uniform bound away from this point.

Theorem 5. Assume that $y=\frac{\sqrt{7}}{12 \sqrt{n}}$, and let $M>0$ be fixed.

1. If $|x| \leqslant M y$, then, as $n \rightarrow \infty$,

$$
f_{1}(q)=\sqrt{-6 i \tau} e^{\frac{7 \pi i}{72 \tau}}+O\left(n^{-\frac{3}{4}} e^{\frac{\pi \sqrt{7 n}}{6}}\right) .
$$


2. If $M y<|x| \leqslant \frac{1}{2}$, then as $n \rightarrow \infty$,

$$
\left|f_{1}(q)\right| \ll n^{\frac{3}{4}} \exp \left(\frac{8 \pi \sqrt{n}}{3 \sqrt{7}}\left(1-\frac{27}{4 \pi^{2}}\left(1-\frac{1}{\sqrt{1+M^{2}}}\right)\right)\right) .
$$

A short calculation shows that the bound in part 2 is indeed an error term as long as $M>\left(\left(\frac{12}{12-\pi^{2}}\right)^{2}-1\right)^{1 / 2}=5.543 \ldots$

Corollary 6. If $M \geqslant 6$ and $M y<|x| \leqslant \frac{1}{2}$, then for some $\varepsilon>0$ we have

$$
f_{1}(q)=O\left(e^{\frac{1}{y}\left(\frac{7 \pi}{72}-\varepsilon\right)}\right)
$$

Proof. In order to determine the behavior of $f_{1}(q)$ near $q=1$, we begin by studying $\bar{\gamma}(q)$ near this point. By Taylor's Theorem, we have

$$
\bar{\gamma}(q)=\bar{\gamma}(1)+O(|\tau|)
$$

and by (3), we directly calculate

$$
\bar{\gamma}(1)=\sum_{k \geqslant 0}\left(\frac{2}{3}\right)^{k}=3 .
$$

Since $|x| \leqslant M y$, we therefore have the following expansion as $n \rightarrow \infty$ :

$$
\bar{\gamma}(q)=3+O\left(n^{-\frac{1}{2}}\right) .
$$

We next determine the asymptotic behavior of the infinite product. The modular inversion formula for Dedekind's eta-function (page 121, Proposition 14 of [10]) implies that, as $\tau \rightarrow 0$,

$$
(q)_{\infty}=\frac{1}{\sqrt{-i \tau}} e^{-\frac{\pi i \tau}{12}-\frac{\pi i}{12 \tau}}\left(1+O\left(e^{-\frac{2 \pi i}{\tau}}\right)\right) .
$$

This formula directly gives that, as $n \rightarrow \infty$,

$$
\frac{\left(q^{3} ; q^{3}\right)_{\infty}}{(q)_{\infty}\left(q^{2} ; q^{2}\right)_{\infty}}=\sqrt{\frac{-2 i \tau}{3}} e^{\frac{7 \pi i}{72 \tau}}\left(1+O\left(e^{-\frac{8 \pi}{\left(M^{2}+1\right)} \sqrt{\frac{n}{7}}}\right)\right) .
$$

Combining (22) and (24) completes the proof of (20).

We next consider $f_{1}(q)$ away from $q=1$. We begin by considering the sum in (19). Bounding each term in the sum absolutely, we get

$$
\left|\sum_{k \geqslant 1} \frac{(-1)^{k} q^{k^{2}+k}}{\left(1-\zeta_{3} q^{k}\right)\left(1-\zeta_{3}^{-1} q^{k}\right)}\right| \ll \frac{1}{(1-|q|)^{2}} \sum_{k \geqslant 1}|q|^{k^{2}} \ll n^{\frac{5}{4}},
$$

where the final bound follows either by an integral comparison or using the transformation law of the theta function. 
Simplifying all of the infinite products from (18) and (19), we have

$$
f_{1}(q)=\frac{\left(q^{3} ; q^{3}\right)_{\infty}}{(q)_{\infty}^{3}} \cdot O\left(n^{\frac{5}{4}}\right)
$$

For convenience denote the above product by $g_{1}(q)$. We calculate

$$
\begin{aligned}
\log \left(g_{1}(q)\right) & =\sum_{m \geqslant 1}\left(\frac{3 q^{m}}{m\left(1-q^{m}\right)}-\frac{q^{3 m}}{m\left(1-q^{3 m}\right)}\right) \\
& =\sum_{m \geqslant 1}\left(\frac{3 q^{3 m-2}}{(3 m-2)\left(1-q^{3 m-2}\right)}+\frac{3 q^{3 m-1}}{(3 m-1)\left(1-q^{3 m-1}\right)}\right) .
\end{aligned}
$$

This implies that

$$
\begin{gathered}
\left|\log \left(g_{1}(q)\right)\right| \leqslant \sum_{m \geqslant 1}\left(\frac{3|q|^{3 m+1}}{(3 m+1)\left(1-|q|^{3 m+1}\right)}+\frac{3|q|^{3 m-1}}{(3 m-1)\left(1-|q|^{3 m-1}\right)}\right) \\
\quad+\frac{3|q|}{|1-q|} \\
=\log \left(g_{1}(|q|)\right)-3|q|\left(\frac{1}{1-|q|}-\frac{1}{|1-q|}\right) .
\end{gathered}
$$

Using the transformation (23), we find that

$$
g_{1}(|q|)=\frac{\eta(3 i y)}{\eta(i y)^{3}}=\frac{y}{\sqrt{3}} e^{\frac{2 \pi}{9 y}}\left(1+O\left(e^{\frac{-2 \pi}{3 y}}\right)\right) .
$$

To bound the other terms, we use the fact that $|x|>M y$, which implies that $\cos (2 \pi x)<$ $\cos (2 \pi M y) \leqslant 1$. Thus

$$
|1-q|^{2}=1-2 e^{-2 \pi y} \cos (2 \pi x)+e^{-4 \pi y}>1-2 e^{-2 \pi y} \cos (2 \pi M y)+e^{-4 \pi y} ;
$$

calculating the Taylor expansion of the final expression (around $y=0$ ) then gives

$$
|1-q|=c_{0}+c_{1} y+O\left(y^{2}\right),
$$

with $c_{0} \geqslant 0$ and $c_{1}>2 \pi \sqrt{1+M^{2}}$. Furthermore, we have

$$
1-|q|=1-e^{-2 \pi y}=2 \pi y+O\left(y^{2}\right) .
$$

Converting (28) and (29) to Laurent series and combining with (26) and (27), we obtain

$$
\left|g_{1}(q)\right| \ll y \exp \left[\frac{1}{y}\left(\frac{2 \pi}{9}-\frac{3}{2 \pi}\left(1-\frac{1}{\sqrt{1+M^{2}}}\right)\right)\right] .
$$

This completes the proof of (21) and Theorem 5 . 


\subsection{Wright's Circle Method and the asymptotic formula for $\bar{s}(n)$}

We now apply Wright's version of the Circle Method [15] and prove the first formula in Theorem 4. By Cauchy's Theorem, we have for all $n \geqslant 1$,

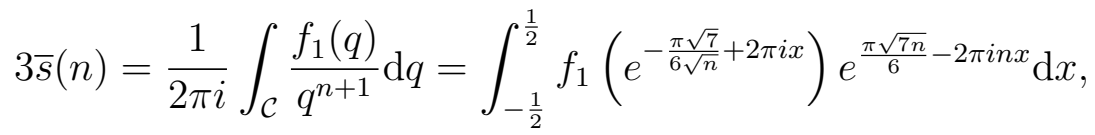

where the contour is the counterclockwise traversal of the circle $\mathcal{C}:=\left\{|q|=e^{-2 \pi y}\right\}$, and $y=\frac{\sqrt{7}}{12 \sqrt{n}}$ as in the previous section. We separate (30) into two integrals, writing $3 \bar{s}(n)=I^{\prime}+I^{\prime \prime}$, where the ranges are split as

$$
I^{\prime}:=\int_{|x| \leqslant M y} \text { and } I^{\prime \prime}:=\int_{M y<|x| \leqslant \frac{1}{2}} .
$$

It turns out that $I^{\prime}$ provides the main asymptotic contribution, while $I^{\prime \prime}$ is an error term.

We approximate $I^{\prime}$ by Bessel functions, which are defined for real $s$ and $u>0$ by

$$
I_{-s-1}(2 u):=\frac{1}{2 \pi i} \int_{\Gamma} t^{s} e^{\pi u\left(t+\frac{1}{t}\right)} \mathrm{d} t,
$$

where $\Gamma$ is Hankel's standard contour that begins in the lower-half plane at $-\infty$, goes counterclockwise around the origin and then goes back to $-\infty$ in the upper-half plane. The Bessel functions have the well-known asymptotic expansion (see (4.12.7) in [2])

$$
I_{\ell}(x)=\frac{e^{x}}{\sqrt{2 \pi x}}+O\left(\frac{e^{x}}{x^{\frac{3}{2}}}\right) .
$$

Assuming that $M>0$ is fixed and that $u>0$ is a real variable, we introduce the auxiliary function

$$
P_{s}(u):=\frac{1}{2 \pi i} \int_{1-M i}^{1+M i} v^{s} e^{u\left(v+\frac{1}{v}\right)} \mathrm{d} v .
$$

Finally, we note a result relating $P_{s}$ to a Bessel function, which is proven in essentially the same manner as Lemma XVII on page 138 of [15].

Lemma $7([15])$. As $n \rightarrow \infty$,

$$
P_{s}=I_{-s-1}(2 u)+O\left(e^{u}\right) .
$$

Proof of (11). Making the change of variables $v=-\frac{i \tau}{y}=-\frac{12 \sqrt{n} i}{\sqrt{7}}$ and using Theorem 1 , we write $I^{\prime}$ as

$$
\begin{aligned}
I^{\prime} & =-i y \int_{1-M i}^{1+M i} f_{1}\left(e^{-\frac{\pi \sqrt{7} v}{6 \sqrt{n}}}\right) e^{\frac{\pi \sqrt{7 n} v}{6}} \mathrm{~d} v \\
& =-i \sqrt{6} y^{\frac{3}{2}} \int_{1-M i}^{1+M i}\left(\sqrt{v} e^{\frac{\pi \sqrt{7}}{6 \sqrt{n} v}}+O\left(n^{-\frac{3}{4}} e^{\frac{\pi \sqrt{7 n}}{6}}\right)\right) e^{\frac{\pi \sqrt{7 n} v}{6}} \mathrm{~d} v .
\end{aligned}
$$


Combining (20), (31), and Lemma 7 (with $u=\frac{\pi \sqrt{7}}{6 \sqrt{n}}$ and $s=\frac{1}{2}$ ), we find that, as $n \rightarrow \infty$,

$$
I^{\prime}=\frac{\sqrt{21}}{12} \frac{e^{\frac{\pi \sqrt{7 n}}{3}}}{n}+O\left(n^{-\frac{5}{4}} e^{\frac{\pi \sqrt{7 n}}{3}}\right) .
$$

We now turn to $I^{\prime \prime}$ and show that it is negligible compared to $I^{\prime}$. Applying Corollary 6 and taking the absolute value of the rest of the integrand, we directly obtain that, for some $\varepsilon>0$,

$$
I^{\prime \prime} \ll e^{\frac{\sqrt{7 n} \pi}{3}(1-\varepsilon)} .
$$

This completes the proof.

\subsection{Asymptotic behavior of $f_{2}(q)$}

In this section we apply Wright's Circle Method to $f_{2}(q)$, where now the dominant cusp is $q=-1$, which can be seen by comparing the singularities of the product $\frac{\left(-q^{3} ; q^{3}\right)_{\infty}}{(-q)_{\infty}^{3}}$. As such, in this section we change the parametrization of the unit disc to $\tau=x+i y$ with $x \in[0,1)$.

Recalling (4) (and [5]), we again provide an alternative series,

$$
\bar{\chi}(q)=\frac{(-q)_{\infty}}{(q)_{\infty}}\left(1+2 \sum_{k \geqslant 1} \frac{(-1)^{k} q^{k^{2}+k}}{\left(1+\zeta_{3} q^{k}\right)\left(1+\zeta_{3}^{-1} q^{k}\right)}\right) .
$$

The asymptotic behavior of $f_{2}(q)$ is recorded in the following result.

Theorem 8. Assume that $y=\frac{1}{6 \sqrt{n}}$, and let $M>0$ be fixed.

1. If $\left|x-\frac{1}{2}\right| \leqslant M y$, then as $n \rightarrow \infty$,

$$
f_{2}(q)=\frac{1}{3} \exp \left(\frac{\pi i}{18\left(\tau-\frac{1}{2}\right)}\right)+O\left(n^{-\frac{1}{2}} e^{\frac{\pi \sqrt{n}}{3}}\right) .
$$

2. If $M y<\left|x-\frac{1}{2}\right| \leqslant \frac{1}{2}$, then as $n \rightarrow \infty$,

$$
\left|f_{2}(q)\right| \ll n \exp \left(\frac{7 \pi \sqrt{n}}{12}\left(1-\frac{36}{7 \pi^{2}}\left(1-\frac{1}{\sqrt{1+M^{2}}}\right)\right)\right) .
$$

Corollary 9. If $M y<\left|x-\frac{1}{2}\right| \leqslant \frac{1}{2}$ and $M \geqslant 6$, then for some $\varepsilon>0$ we have

$$
f_{2}(q)=O\left(e^{\frac{1}{y}\left(\frac{\pi}{18}-\varepsilon\right)}\right) .
$$


Proof. A direct calculation using (4) shows that $\bar{\chi}(-1)=\frac{1}{3}$, so that its Taylor expansion satisfies $\bar{\chi}(q)=\frac{1}{3}+O\left(n^{-\frac{1}{2}}\right)$. To determine the asymptotic behavior of the product $\frac{\left(-q^{3} ; q^{3}\right)_{\infty}}{(-q)_{\infty}^{3}}$ near $q=-1$, we make the change of variables $w:=\tau-\frac{1}{2}=x-\frac{1}{2}+i y$, and correspondingly $Q:=e^{2 \pi i w}=-q$. Denote the product under this change of variables by

$$
h_{2}(Q):=\prod_{m \geqslant 1} \frac{\left(1+\left(-Q^{3}\right)^{m}\right)}{\left(1+(-Q)^{m}\right)^{3}}=\frac{\left(Q^{2} ; Q^{2}\right)_{\infty}^{6}\left(Q^{3} ; Q^{3}\right)_{\infty}\left(Q^{12} ; Q^{12}\right)_{\infty}}{(Q)_{\infty}^{3}\left(Q^{4} ; Q^{4}\right)_{\infty}^{3}\left(Q^{6} ; Q^{6}\right)_{\infty}^{2}} .
$$

Applying (23) implies that

$$
h_{2}(Q)=e^{\frac{\pi i}{18 w}}\left(1+O\left(e^{-\frac{\pi i}{6 w}}\right)\right)
$$

This gives (34)

Next, using a similar argument as in (25), we conclude that

$$
\left|\sum_{k \geqslant 1} \frac{(-1)^{k} q^{k^{2}+k}}{\left(1+\zeta_{3} q^{k}\right)\left(1+\zeta_{3}^{-1} q^{k}\right)}\right| \ll n^{\frac{5}{4}} .
$$

Combining the products from (6) and (33), we therefore write

$$
g_{2}(q):=\frac{\left(-q^{3} ; q^{3}\right)_{\infty}}{(q)_{\infty}(-q ; q)_{\infty}^{2}}=\frac{(q)_{\infty}\left(q^{6} ; q^{6}\right)_{\infty}}{\left(q^{2} ; q^{2}\right)_{\infty}^{2}\left(q^{3} ; q^{3}\right)_{\infty}} .
$$

After some simplification, we obtain

$$
\log \left(g_{2}(q)\right)=\sum_{m \geqslant 1}\left(-\frac{q^{m}}{m\left(1+q^{m}\right)}+\frac{q^{3 m}}{m\left(1-q^{6 m}\right)}\right) .
$$

We therefore have the bound

$$
\begin{aligned}
\left|\log \left(g_{2}(q)\right)\right| & \leqslant \sum_{m \geqslant 1}\left(\frac{|q|^{m}}{m\left(1-|q|^{m}\right)}+\frac{|q|^{3 m}}{m\left(1-|q|^{\mid 6 m}\right)}\right)-|q|\left(\frac{1}{1-|q|}-\frac{1}{|1+q|}\right) \\
& =\log \left(\frac{\left(|q|^{6} ;|q|^{6}\right)_{\infty}}{(|q| ;|q|)_{\infty}\left(|q|^{3} ;|q|^{3}\right)_{\infty}}\right)-|q|\left(\frac{1}{1-|q|}-\frac{1}{|1+q|}\right) .
\end{aligned}
$$

Once again (23) implies that

$$
\frac{\left(|q|^{6} ;|q|^{6}\right)_{\infty}}{(|q| ;|q|)_{\infty}\left(|q|^{3} ;|q|^{3}\right)_{\infty}}=\sqrt{\frac{y}{2}} e^{\frac{7 \pi}{72 y}}(1+o(y)) .
$$

Now we use the fact that $\left|x-\frac{1}{2}\right|>M y$, which implies that

$$
\cos (2 \pi x)=\cos (2 \pi|x|)=-\cos \left(2 \pi\left(\frac{1}{2}-x\right)\right)>-\cos (2 \pi M y)
$$


from which we conclude

$$
|1+q|^{2}=1+2 e^{-2 \pi y} \cos (2 \pi x)+e^{-4 \pi y}>1-2 e^{-2 \pi y} \cos (2 \pi M y)+e^{-4 \pi y} .
$$

The remainder of the proof of (35) proceeds analogously to the arguments following equation (28).

Proof of (12). We proceed as in Section 4.2, with the main differences due to the different parameterization around $q=-1$. By Cauchy's Theorem, we again have that for all $n \geqslant 1$,

$$
\bar{t}_{+}(n)-\bar{t}_{-}(n)=\frac{1}{2 \pi i} \int_{\mathcal{C}} \frac{f_{2}(q)}{q^{n+1}} \mathrm{~d} q=\int_{0}^{1} f_{2}\left(e^{-2 \pi y+2 \pi i x}\right) e^{2 \pi n y-2 \pi i n x} \mathrm{~d} x,
$$

where the contour is the counterclockwise traversal of the circle $\mathcal{C}:=\left\{|q|=e^{-2 \pi y}\right\}$, and $y=\frac{1}{6 \sqrt{n}}$ as above. We now split the integral as

$$
I^{\prime}:=\int_{\left|x-\frac{1}{2}\right| \leqslant M y} \text { and } I^{\prime \prime}:=\int_{\left|x-\frac{1}{2}\right|>M y},
$$

and reparameterize $I^{\prime}$ using $\tilde{x}=x-\frac{1}{2}$ (and again also writing $\tilde{\tau}=\tau-\frac{1}{2}$ ). After applying Theorem 1, we obtain

$$
I^{\prime}=\int_{|\tilde{x}| \leqslant M y} f_{2}\left(e^{2 \pi i \tau}\right) e^{2 \pi n\left(\tilde{\tau}+\frac{1}{2}\right)} \mathrm{d} \tilde{x}=\int_{|\tilde{x}| \leqslant M y}\left(\frac{1}{3} e^{\frac{\pi i}{18 \tilde{\tau}}}+O\left(n^{-\frac{1}{2}} e^{\frac{\pi \sqrt{n}}{3}}\right)\right) e^{2 \pi n \tilde{\tau}}(-1)^{n} \mathrm{~d} \tilde{x} .
$$

After pulling the $(-1)^{n}$ out of the integral, the remainder of the argument follows as in (32) (note that Lemma 7 is now applied with $u=\frac{\pi \sqrt{n}}{3}$ and $s=0$ ).

\section{Concluding Remarks}

We close by discussing the relationship between the combinatorial functions studied in this paper and Ramanujan's classical mock theta functions, as well as some applications of similar series. For $\ell \in \mathbb{N}$ we define

$$
f_{2, \ell}(x):=f_{2, \ell}(x ; q):=1+\sum_{n=1}^{\infty} \frac{x^{n} q^{n}}{(q)_{n}} \prod_{j=0}^{n-1}\left(1+(\ell-1) q^{j}+q^{2 j}\right) .
$$

If we further set

$$
f_{2, \ell}^{*}(q):=\frac{(q)_{\infty}}{\prod_{k \geqslant 1}\left(1+(\ell-1) q^{k}+q^{2 k}\right)} \cdot f_{2, \ell}(1)
$$


then we have the following relations to mock theta functions, using the notation of [14]:

$$
\begin{aligned}
\chi(q) & =f_{2,0}^{*}(q), \\
\phi(q) & =f_{2,1}^{*}(q), \\
\gamma(q) & =f_{2,2}^{*}(q), \\
f(q) & =f_{2,3}^{*}(q) .
\end{aligned}
$$

Moreover, for $\ell=0$ or 1 , the function $f_{2, \ell}(x)$ has recently played a role in studies of partitions without sequences [3] and lower 1-run overpartitions [4].

The function $\bar{K}(x ; q)$ in Section 2 of the present paper is the case $\ell=0$ of a similar function,

$$
\bar{f}_{2, \ell}(x):=\bar{f}_{2, \ell}(x ; q):=1+\sum_{n=1}^{\infty} \frac{x^{n} q^{n}}{\left(q^{2} ; q^{2}\right)_{n}} \prod_{j=0}^{n-1}\left(1+(\ell-1) q^{j}+q^{2 j}\right) .
$$

Both $f_{2, \ell}$ and $\bar{f}_{2, \ell}$ satisfy simple $q$-difference equations, namely

$$
f_{2, \ell}(x)=\frac{(1+(\ell-1) x q)}{1-x q} f_{2, \ell}(x q)+\frac{x q}{1-x q} f_{2, \ell}\left(x q^{2}\right)
$$

and

$$
\bar{f}_{2, \ell}(x)=\frac{(\ell-1) x q}{1-x q} \bar{f}_{2, \ell}(x q)+\frac{(1+x q)}{1-x q} \bar{f}_{2, \ell}\left(x q^{2}\right) .
$$

It would be worthwhile to investigate these series for other $\ell$. In particular, if $\ell=0,1,2$, or 3 , then the functions $\bar{f}_{2, \ell}(1)$ are related to mock theta functions in [5] via (17).

\section{References}

[1] G.E. Andrews, The theory of partitions, Cambridge University Press, Cambridge, 1998.

[2] G.E. Andrews, R. Askey, and R. Roy, Special Functions, Encyclopedia of Mathematics and its Applications, Cambridge University Press, 2001.

[3] G.E. Andrews, Partitions with short sequences and mock theta functions, Proc. Natl. Acad. Sci. USA 102 (2005), no. 13, 4666-4671.

[4] K. Bringmann, A. Holroyd, K. Mahlburg, and M. Vlasenko, $k$-run overpartitions and mock theta functions, Q. J. Math. 64 (2013), no. 4, 1009-1021.

[5] K. Bringmann and J. Lovejoy, Dyson's rank, overpartitions, and weak Maass forms, Int. Math. Res. Not. (2007), rnm063.

[6] K. Bringmann and J. Lovejoy, Overpartitions and class numbers of binary quadratic forms, Proc. Natl. Acad. Sci. USA 106 (2009), no. 14, 5513-5516.

[7] K. Bringmann, J. Lovejoy, and R. Osburn, Rank and crank moments for overpartitions, J. Number Theory 129 (2009), 1758-1772. 
[8] G. Gasper and M. Rahman, Basic Hypergeometric Series, second edition, Cambridge University Press, Cambridge, 2004.

[9] G. Hardy and S. Ramanujan, Asymptotic formulae for the distribution of integers of various types, Proc. London Math. Soc. (2) 16 (1918), 112-32.

[10] N. Koblitz, Introduction to elliptic curves and modular forms, Graduate Texts in Mathematics 97, Springer-Verlag, New York, 1984.

[11] J. Lovejoy and R. Osburn, Mixed mock modular q-series, J. Indian Math. Soc. (N.S), New Series, Special volume to commemorate the 125th birth anniversary of Srinivasa Ramanujan (2013), 45-61.

[12] H. Rademacher, The Fourier Coefficients of the Modular Invariant $J(\tau)$, Amer. J. Math. 60 (1938), 501-512.

[13] H. Rademacher and H. Zuckerman, On the Fourier coefficients of certain modular forms of positive dimension, Ann. of Math. 39 (1938), 433-462.

[14] G. Watson, The final problem : An account of the mock theta functions, J. London Math. Soc. S1-11 (1936), 55-80.

[15] E. Wright, Asymptotic partition formulae II. Weighted partitions, Proc. London Math. Soc. (2) 36 (1933), 117-141. 